\title{
Combining Ability Studies for Development of New Hybrids in Rice over Environments
}

\author{
P. Saidaiah (Corresponding Author) \\ College of Horticulture, Andhra Pradesh Horticultural University \\ 1 st floor of SBH, H.No.15-157, Kurnool Road, Kothakota (Post)-509381 \\ Mahaboobnagar (dist), Andhrapradesh, India \\ Tel: 91-996-342-9499 E-mail: saidu_genetics@yahoo.co.in \\ S. Sudheer kumar \\ Department of Genetics \& Plant Breeding \\ College of Agriculture, Acharya N.G.Ranga Agricultural University \\ Rajendra Nagar, Hyderabad-500 030, Andhra Pradesh, India \\ Tel: 91-939-109-1071 E-mail: sagisudheer@yahoo.com
}

M.S. Ramesha

Senior Project Investigator, Cereals System Initiative for South Asia (CSISA)

IRRI Regional Office, Barwale Research Foundation, Banjarahills

Hyderabad-500 031, Andhra Pradesh, India

Tel: 91-944-149-6778Ｅ-mail: mugalodimsr@yahoo.com

\begin{abstract}
Combining ability study on grain yield and its components from line $\mathrm{x}$ tester analysis over the locations of five well adapted CMS lines and twenty three testers of different eco-geographic origin revealed higher SCA variance than $G C A$ variance for all the traits indicating the prevalence of non-additive gene action. The lines APMS 6A, PUSA 5A and CRMS 32A and testers 1096, 1005, IBL-57 and SC5 9-3 were the good general combiners for yield and its majority of the traits. IBL-57 was the only good general combiner among the male parents for earliness, dwarfness and grain yield per plant. The hybrids APMS 6A x SC5 9-3, APMS 6A x 1005 and APMS $6 \mathrm{~A} \times \mathrm{GQ} 25$ were identified as potential one for yield and desired traits based on $s c a$ effects.
\end{abstract}

Keywords: Hybrid rice, CMS lines, Combining ability, Gene action

\section{Introduction}

The development and use of hybrid rice varieties on commercial scale utilizing CMS - fertility restoration system has proved to be one of the mile stones in the history of rice improvement. The hybrid rice technology now in operation, aims at yield increment through higher exploitable heterosis levels. With increasing interest in exploitation of heterosis in rice, there is an urgent need to subject various CMS lines and restorer lines for combining ability tests. The knowledge of combining ability is useful to assess nicking ability in self pollinated crops and at the same time elucidate the nature and magnitude of gene actions involved. It provides to the breeders an insight in to nature and relative magnitude of fixable and non-fixable genetic variances. Therefore, the present investigation was carried out to estimate combining ability effects for yield and its components involving CMS lines and restorer lines in rice.

\section{Material and Methods}

The material for the present study comprised $115 \mathrm{~F}_{1} \mathrm{~s}$ of rice generated involving five CMS lines (viz., IR 58025A, IR 79156A, APMS 6A, PUSA 5A and CRMS 32A) and 23 diverse elite restorer lines as testers through Line $\mathrm{x}$ Tester design during rabi, 2006-07. The resultant $115 \mathrm{~F}_{1} \mathrm{~s}$ and 28 parents were grown in randomized complete block design with two replications during kharif, 2007 at three different locations viz., Directorate of 
Rice Research, Hyderabad (Southern Telangana Agro-climatic zone), Regional Agricultural Research Station, Warangal (Central Telangana Agro-climatic zone) and Regional Agricultural Research Station, Jagtial (Northern Telangana Agro-climatic zone. Thirty days old seedlings were transplanted with one seedling per hill adopting $20 \times 15 \mathrm{~cm}$ spacing and each entry was planted in two rows of $1.8 \mathrm{~m}$ length. All the recommended agronomic practices were followed. In each entry, five plants were selected randomly from each replication and biometrical observations were recorded for plant height, flag leaf length, flag leaf width, productive tillers per plant, panicle length, panicle weight, filled grains per panicle, spikelet fertility \%, 1000 grain weight, grain yield per plant and productivity per day. Days to 50\% flowering was recorded on plot basis. The mean data over the three locations were analyzed for combining ability following the standard method of Kempthorne (1957).

\section{Results and Discussion}

The pooled analysis of variance for combining ability over three locations revealed that the variance due to parents, parents vs. crosses and crosses were highly significant (Table 1) for all the characters studied. The variance due to lines was significant for all the characters except days to fifty per cent flowering, plant height and 1000 grain weight, whereas for testers, plant height, flag leaf length, productive tillers per plant, panicle length, filled grains per panicle and 1000 grain weight were found significant. Interaction effects of (Parents vs. crosses) $\mathrm{x}$ locations, parents $\mathrm{x}$ locations and crosses $\mathrm{x}$ locations were significant for all the characters, except flag leaf width in case of (parents vs. crosses) $\mathrm{x}$ locations interaction. Further partitioning of crosses $\mathrm{x}$ locations indicated that the interaction of lines $\mathrm{x}$ locations showed significant differences for productive tillers per plant, flag leaf length and flag leaf width, while testers $x$ locations was significant only for filled grains per panicle. The interaction due to lines $\mathrm{x}$ testers and lines $\mathrm{x}$ testers $\mathrm{x}$ locations were significant for all the traits indicating that combining ability contributed heavily in the expression of these traits and provided importance of dominance or non additive variances for all the traits. The comparative estimates of variances due to $G C A$ and $S C A$ revealed the importance of $S C A$ variance. The $S C A$ variances were higher than $G C A$ variances for all the traits suggesting the significant role of non additive gene action Predominance of non additive gene action for grain yield and its components was also reported by other workers (Satyanarayana et al.(2000), Rita and Motiramani (2005) ; Singh et al. (2005); Venkatesan et al. (2007) and Dalvi and Patel (2009) )

The estimates of $g c a$ effects (Table 2) showed that parents with high $g c a$ effects differed for various traits. Among the lines APMS 6A had favourable genes for grain yield and other traits including flag leaf length, flag leaf width, productive tiller per plant, panicle weight, filled grains per panicle, spikelet fertility $\%$ and productivity per day. The line PUSA 5A was the best combiner for grain yield, dwarfness, 1000 seed weight and productivity per day. However CRMS 32A contributed positive alleles for grain yield, dwarfness, panicle length, panicle weight, filled grains per panicle and productivity per day. Among the testers, 1096 possessed the desirable genes for grain yield, productive tillers per plant, panicle length, panicle weight, filled grains per panicle, and productivity per day, whereas 1005 for grain yield, dwarfness, flag leaf length, flag leaf width, panicle weight, filled grains per panicle and productivity per day. Some other good general combiners that had also contributed positive genes for various characters were; IBL-57 for grain yield, earliness of flowering, dwarfness, flag leaf length, productive tillers per plant, filled grains per panicle and productivity per day; SC5 9-3 for grain yield, flag leaf length, flag leaf width, productive tillers per plant, filled grains per panicle and spikelet fertility $\%$ and 124 for grain yield, panicle length, panicle weight, filled grains per panicle, spikelet fertility $\%$ and productivity per day.

Crosses with desirable $s c a$ effects for various traits along with mean performance and $g c a$ effects of parents involved in the crosses are listed in the table 3. The crosses APMS 6A X SC5 9-3, APMS 6A X 1005 and APMS 6A X GQ 25, PUSA 5A X IR 55 and PUSA 5A X IR 43 expressed significant sca effects as well as high per se performance for grain yield per plant and few other traits. However, CRMS 32A X IR 43 and PUSA 5A X IR 60 for plant height (dwarfness), IR 79156A X GQ-120 for flag leaf length, APMS 6A X 118 for flag leaf width, APMS 6A X SC 5 9-3 and APMS 6A X 118 for panicle weight, APMS 6A X 1005 for filled grains per panicle, APMS 6A X SC5 9-3 and APMS 6A X 1005 for grain yield per plant, PUSA 5A X KMR 3 for productivity per day had high mean performance and highly significant mean values. These desirable cross combinations involved high $\mathrm{x}$ high type of general combiners. Kalitha and Upadhaya (2000), Shivani et al. (2009) and Salgotra et al. (2009) also reported about interaction between positive and positive alleles in crosses involving high $\mathrm{x}$ high combiners which can be fixed in subsequent generations if no repulsion phase linkages are involved.

The desirable performance of combinations like high $\mathrm{x}$ low may be ascribed to the interaction between dominant alleles from good combiners and recessive alleles from poor combiners (Dubey, 1975). Such combinations were observed in the hybrids ; IR 58025AX BR 827-35 and IR 58025A X EPLT 109 for days to 50\% flowering (earliness), IR 79156A X GQ 25 and IR 79156A X EPLT 109 for plant height (dwarfness), CRMS 32A X SC5 
9-3, CRMS 32A X IBL-57 and IR 79156A x 1096 for flag leaf length, APMS 6A X 619-2 for flag leaf width, CRMS 32A X IR 43 for productive tillers per plant, IR 58025A XGQ 37-1, APMS 6A X GQ 120, IR 79156A X GQ 70 and IR 58025A X 517 for panicle length, IR 79156A X SG 27-77 for filled grains per panicle, APMS 6A X 611-1 for spikelet fertility \%, PUSA 5A X 517, APMS 6A X IR 60 and APMS 6A X EPLT 109 for 1000 grain weight, APMS 6A X GQ 25, PUSA 5A X IR 43 and PUSA 5A XIR 55 for grain yield per plant and APMS 6A X GQ 25, PUSA 5A X IR 43, PUSA 5A X IR 55 and IR 79156A X IBL 57 for productivity per day. Peng and Virmani (1990) also reported the possibility of interaction between positive alleles from good combiner and negative alleles from poor combiner in high $\mathrm{x}$ low cross combination and suggested for the exploitation of $\mathrm{F}_{1}$ generation, as their high yielding potential would be unfixable in succeeding generation.

Involvement of both the poor combiners also produced superior specific combining hybrids as evidenced from the combinations: PUSA 5A X IR 60, CRMS 32A X IR 43 and APMS 6A X 118 (days to 50\% flowering (earliness), IR 79156A X GQ-120 (plant height (dwarfness)), CRMS 32 A X 118 (flag leaf length), PUSA 5A X GQ 37-1, CRMS 32A X 124 and CRMS 32A X IBL 57 (flag leaf width), PUSA 5A X 1096, IR 79156A X KMR 3, APMS 6A X SC 5 9-3 and APMS 6A X124 (productive tillers per plant), PUSA 5A X SC5 2-2-1(panicle length), IR 58025A X IR 60, IR 79156A X SG 27-77 and IR 79156A X GQ 37-1 (panicle weight), PUSA 5A X 619-2 and PUSA 5A X 611-1 (filled grains per panicle), PUSA 5A X 611-1, CRMS 32A X EPLT-109 and IR 58025A X IR 55 (spikelet fertility \%) and APMS 6A X IBL 57 and CRMS 32A X IR 43 (1000 grain weight). Involvement of both the combiners with low $g c a$ has been attributed to over dominance and epistasis interaction, which has been suggested by Amrithadevarathinam (1983), Singh et al. (2005) and Dalvi and Patel (2009). In majority of the crosses, high sca was mainly either due to high $\mathrm{x}$ low or low $\mathrm{x}$ low combining parents, which further substantiate the operation of non-additive gene action (additive $\mathrm{x}$ dominance and dominance $\mathrm{x}$ dominance epistatic interaction).

Combining ability analysis revealed that 1096, 1005, IBL-57 and SC5 9-3 were the good general combiners for yield and its majority of the trait among the male lines, whereas APMS 6A, PUSA 5A and CRMS 32A were among female lines. APMS 6A x SC5 9-3, APMS 6A x 1005 and APMS 6A x GQ 25 were identified as most promising crosses for yield based on $s c a$ effects, better per se and both or one of the parents with high gca for yield per plant also, could be exploited profitably for yield in rice.

\section{References}

Amrithadevarathinam, A. (1983). Combining ability and heterosis in dry and semi-dry paddy. Madras Agricultural Journal, 70, 233-237.

Dalvi, V.V. \& Patel, D.V. (2009). Combining ability anlysis for yield in hybrid rice. Oryza, 46 (2), 97-102

Dubey, R.S. (1975). Combining ability for cigar filler tobacco. Indian Journal of Genetics and Plant Breeding, 75, 76-82.

Kalitha, U.C. \& Upadhaya, L. P. (2000). Line x Tester analysis of combining ability in rice under irrigated low land condition. Oryza, 37, 15-19.

Kempthorne, O. (1957). An introduction to genetic statistics. John Wiley and Sons, Inc: New York.

Peng, J.Y. \& Virmani, S.S. (1990). Combining ability for yield and yield related traits in relation to breeding in rice. Oryza, 27, 1-10.

Rita, B. \& Motiramani, N.K. (2005). Study on gene action and combining ability in rice. Oryza, 42, 153-155

Salgotra, R.K., Gupta, B.B. \& Praveen Singh. (2009). Combining ability studies for yield and yield components in basmati rice. Oryza, 46 (1), 12-16.

Satyanarayana, P.V., Reddy, M.S.S., Ish Kumar \& Madhuri, J. (2000). Combining ability studies on yield and yield components in rice. Oryza, 37, 22-25.

Shivani, D., Viraktamath, B.C. \& Shobha Rani, N. (2009). Combining ability for yield and grain quality characters in indica/indica hybrids of rice. Oryza, 46 (2), 152-155.

Singh, R.V., Maurya, D.M., Dwivedi, J.L. \& Verma, O.P. (2005). Combining ability studies on yield and its components using CMS lines in rice (Oryza sativa L.). Oryza, 42, 306-309.

Venkatesan, M. Anbuselvam, Y., Elangaimannan, R. \& Karthikeyan, P. (2007). Combining ability for yield and physiological characters in rice. Crop Improvement, 44 (4), 296-299. 
Table 1a. Pooled analysis of variance for combining ability (L X T) for yield and yield components in rice

\begin{tabular}{|c|c|c|c|c|c|c|c|}
\hline & \multicolumn{7}{|c|}{ Mean sum of squares } \\
\hline Source of variation & d.f & $\begin{array}{l}\text { Days to } 50 \% \\
\text { flowering }\end{array}$ & Plant height & $\begin{array}{l}\text { Flag leaf } \\
\text { length }\end{array}$ & $\begin{array}{l}\text { Flag leaf } \\
\text { width }\end{array}$ & $\begin{array}{l}\text { Productive } \\
\text { tillers/plant }\end{array}$ & Panicle length \\
\hline Locations & 2 & $8166.540 * *$ & $6484.604 * *$ & $\begin{array}{l}6451.716 \\
* *\end{array}$ & $6.618 * *$ & $86.747 * *$ & $202.405 * *$ \\
\hline $\begin{array}{l}\text { Replications x } \\
\text { Locations }\end{array}$ & 2 & 4.938 & 29.056 & 0.375 & $0.134 * *$ & 0.175 & 0.379 \\
\hline Treatments & 142 & $63.697 * *$ & $336.490 * *$ & $80.313 * *$ & $0.175 * *$ & $12.895 * *$ & $11.118 * *$ \\
\hline Parents & 27 & $105.061 * *$ & $805.453 * *$ & $81.219 * *$ & $0.256 * *$ & $2.885 * *$ & $10.987 * *$ \\
\hline Parent vs. Crosses & 1 & $42.894 * *$ & $1930.397 * *$ & $\begin{array}{l}1593.886 \\
* *\end{array}$ & $0.203 * *$ & $1058.221 * *$ & $514.087 * *$ \\
\hline Crosses & 114 & $54.083 * *$ & $211.438 * *$ & $66.821 * *$ & $0.155 * *$ & $6.097 * *$ & $6.737 * *$ \\
\hline Lines & 4 & 39.357 & 394.483 & $245.108 * *$ & $0.870 * *$ & $10.618 *$ & $19.280 * *$ \\
\hline Testers & 22 & 71.607 & $372.022 * *$ & $109.044 * *$ & 0.189 & $13.195 * *$ & $10.127 *$ \\
\hline Lines $\mathrm{x}$ Testers & 88 & $50.371 * *$ & $162.972 * *$ & $48.162 * *$ & $0.114 * *$ & $4.116 * *$ & $5.319 * *$ \\
\hline Parents x Locations & 54 & $33.297 * *$ & $92.719 * *$ & $40.486 * *$ & $0.095 * *$ & $2.260 * *$ & $3.337 * *$ \\
\hline $\begin{array}{l}\text { (Parent vs. Crosses) } \\
\text { x Locations }\end{array}$ & 2 & $597.974 * *$ & $858.389 * *$ & $81.341 * *$ & 0.030 & $26.055 * *$ & $37.831 * *$ \\
\hline $\begin{array}{l}\text { Crosses x } \\
\text { Locations }\end{array}$ & 228 & $54.380 * *$ & $87.636 * *$ & $42.938 * *$ & $0.079 * *$ & $4.269 * *$ & $2.818 * *$ \\
\hline Lines x Locations & 8 & 42.079 & 130.372 & $200.827 * *$ & $0.289 * *$ & $10.170 *$ & 1.735 \\
\hline Testers $\mathrm{x}$ Locations & 44 & 65.061 & 91.173 & 47.957 & 0.090 & 4.533 & 2.515 \\
\hline $\begin{array}{l}\text { Lines } \mathrm{x} \text { Testers } \mathrm{x} \\
\text { Locations }\end{array}$ & 176 & $52.269 * *$ & $84.809 * *$ & $34.506 * *$ & $0.067 * *$ & $3.935 * *$ & $2.943 * *$ \\
\hline Error & 426 & 3.812 & 9.963 & 8.677 & 0.021 & 0.967 & 0.796 \\
\hline$\sigma^{2} \mathrm{GCA}$ & & 0.61 & 4.44 & 2 & 0.01 & 0.13 & 0.17 \\
\hline$\sigma^{2} \mathrm{SCA}$ & & 7.73 & 25.48 & 6.56 & 0.02 & 0.52 & 0.76 \\
\hline$\sigma^{2} \mathrm{GCA} / \sigma^{2} \mathrm{SCA}$ & & 0.08 & 0.17 & 0.30 & 0.50 & 0.25 & 0.22 \\
\hline
\end{tabular}

$*$ : Significant at $5 \%$ level and $* *$ : Significant at $1 \%$ level 
Table 1b. Pooled analysis of variance for combining ability (L X T) for yield and yield components in rice

\begin{tabular}{|c|c|c|c|c|c|c|c|}
\hline \multicolumn{8}{|c|}{ Mean Sum of Squares } \\
\hline $\begin{array}{l}\text { Source of } \\
\text { variation }\end{array}$ & d.f & $\begin{array}{l}\text { Panicle } \\
\text { weight }\end{array}$ & $\begin{array}{l}\text { Filled grains } \\
\text { per panicle }\end{array}$ & $\begin{array}{l}\text { Spikelet } \\
\text { fertility } \%\end{array}$ & $\begin{array}{l}1000 \text { grain } \\
\text { weight }\end{array}$ & $\begin{array}{l}\text { Grain yield per } \\
\text { plant }\end{array}$ & $\begin{array}{l}\text { Productivity } \\
\text { per day }\end{array}$ \\
\hline Locations & 2 & $10.027 * *$ & $12805.560 * *$ & $4297.021 * *$ & $34.324 * *$ & $876.645 * *$ & $4823.378 * *$ \\
\hline $\begin{array}{l}\text { Replications x } \\
\text { Locations }\end{array}$ & 2 & 0.268 & 160.089 & 0.282 & 0.221 & 1.522 & 12.162 \\
\hline Treatments & 142 & $3.027 * *$ & $7583.527 * *$ & $119.148 * *$ & $25.561 * *$ & $160.233 * *$ & $714.219 * *$ \\
\hline Parents & 27 & $1.932 * *$ & $6297.925 * *$ & $79.294 * *$ & $40.342 * *$ & $52.871 * *$ & $240.599 * *$ \\
\hline $\begin{array}{l}\text { Parent vs. } \\
\text { Crosses }\end{array}$ & 1 & $98.811 * *$ & $100092.800 * *$ & $375.922 * *$ & $51.517 * *$ & $4458.350 * *$ & $20275.250 * *$ \\
\hline Crosses & 114 & $2.446 * *$ & $7076.527 * *$ & $126.335 * *$ & $21.832 * *$ & $147.958 * *$ & $654.804 * *$ \\
\hline Lines & 4 & $21.533 * *$ & $49802.710 * *$ & $312.097 *$ & 21.425 & $503.279 * *$ & $1960.378 *$ \\
\hline Testers & 22 & 2.359 & $9377.320 * *$ & 165.686 & $54.209 * *$ & 156.382 & 711.171 \\
\hline Lines $\mathrm{x}$ Testers & 88 & $1.600 * *$ & $4559.229 * *$ & $108.053 * *$ & $13.756 * *$ & $129.701 * *$ & $581.368 * *$ \\
\hline $\begin{array}{l}\text { Parents x } \\
\text { Locations }\end{array}$ & 54 & $0.829 * *$ & $1856.914 * *$ & $60.818 * *$ & $9.070 * *$ & $30.227 * *$ & $139.273 * *$ \\
\hline $\begin{array}{l}\text { (Parent vs. } \\
\text { Cross) } \mathrm{x} \\
\text { Locations }\end{array}$ & 2 & $3.983 * *$ & $13293.420 * *$ & $104.211 * *$ & $42.976 * *$ & $150.846 * *$ & $545.955 * *$ \\
\hline $\begin{array}{l}\text { Crosses x } \\
\text { Locations }\end{array}$ & 228 & $0.935 * *$ & $2449.021 * *$ & $98.394 * *$ & $13.826 * *$ & $68.936 * *$ & $319.323 * *$ \\
\hline $\begin{array}{l}\text { Lines } \mathrm{x} \\
\text { Locations }\end{array}$ & 8 & 1.043 & 3146.987 & 190.423 & 17.752 & 82.201 & 357.870 \\
\hline $\begin{array}{l}\text { Testers } \mathrm{x} \\
\text { Locations }\end{array}$ & 44 & 1.131 & $2587.760 * *$ & 85.863 & 16.059 & 62.670 & 297.621 \\
\hline $\begin{array}{l}\text { Lines x Testers } \\
\mathrm{x} \text { Locations }\end{array}$ & 176 & $0.882 * *$ & $2382.610 * *$ & $97.344 * *$ & $13.089 * *$ & $69.899 * *$ & $322.996 * *$ \\
\hline Error & 426 & 0.152 & 138.77 & 6.25 & 0.39 & 3.68 & 16.50 \\
\hline$\sigma^{2} \mathrm{GCA}$ & & 0.14 & 350.53 & 2.77 & 0.46 & 3.88 & 15.69 \\
\hline$\sigma^{2} \mathrm{SCA}$ & & 0.24 & 735.69 & 17.02 & 2.23 & 20.97 & 93.98 \\
\hline$\sigma^{2} \mathrm{GCA} / \sigma^{2} \mathrm{SCA}$ & & 0.58 & 0.48 & 0.16 & 0.21 & 0.19 & 0.17 \\
\hline
\end{tabular}

*: Significant at 5\% level and **: Significant at 1\% level 
Table 2. General combining ability (GCA) effects of parents in rice

\begin{tabular}{|c|c|c|c|c|c|c|}
\hline & FLL & FLW & DFF & PHT & EBT & PL \\
\hline \multicolumn{7}{|l|}{ LINES } \\
\hline IR $58025 \mathrm{~A}$ & $-0.73 * *$ & $-0.043 * *$ & $-0.78 * *$ & $1.00 * *$ & $-0.42 * *$ & $0.26 * *$ \\
\hline IR79156A & $1.26 * *$ & $-0.10 * *$ & -0.08 & $1.68 * *$ & -0.06 & $0.20 * *$ \\
\hline APMS 6A & $1.54 * *$ & $0.12 * *$ & $0.63 * *$ & $0.57 *$ & $0.33 * *$ & -0.01 \\
\hline PUSA 5A & $-1.51 * *$ & 0.00 & -0.11 & $-2.63 * *$ & 0.02 & $-0.64 * *$ \\
\hline CRMS32A & $-0.56 *$ & 0.02 & $0.34 *$ & $-0.61 *$ & 0.14 & $0.20 * *$ \\
\hline SE (lines) & 0.25 & 0.01 & 0.17 & 0.27 & 0.09 & 0.07 \\
\hline \multicolumn{7}{|l|}{ Testers } \\
\hline 1096 & 1.05 & $-0.08 * *$ & -0.69 & $3.50 * *$ & $0.88 * *$ & $0.41 * *$ \\
\hline 1005 & $1.12 *$ & $0.12 * *$ & $0.84 *$ & $-1.89 * *$ & 0.10 & $-0.43 * *$ \\
\hline $619-2$ & -0.29 & 0.02 & $0.84 *$ & 0.297 & 0.16 & $0.42 * *$ \\
\hline $612-1$ & 0.09 & 0.02 & $-1.56 * *$ & $3.80 * *$ & $0.92 * *$ & $0.89 * *$ \\
\hline 611-1 & $2.77^{* *}$ & 0.04 & -0.06 & 0.49 & $-0.55 * *$ & 0.28 \\
\hline GQ-25 & -0.87 & $0.06 *$ & 0.31 & $-1.192 *$ & 0.18 & -0.21 \\
\hline GQ-37-1 & -0.82 & 0.05 & $-1.46 * *$ & -0.847 & $0.96 * *$ & $-0.38 *$ \\
\hline GQ-70 & $2.05 * *$ & $0.09 * *$ & $-2.19 * *$ & $-3.46 * *$ & 0.22 & -0.23 \\
\hline GQ-120 & $1.86 * *$ & $-0.06 *$ & 0.01 & $3.51 * *$ & -0.03 & $0.41 *$ \\
\hline KMR-3 & -0.59 & $-0.15 * *$ & $-2.49 * *$ & $5.79 * *$ & $0.80 * *$ & $0.47 * *$ \\
\hline IBL-57 & $2.61 * *$ & -0.04 & $-3.49 * *$ & $-2.277 * *$ & $0.59 * *$ & $-1.19^{* *}$ \\
\hline BR827-35 & 0.62 & 0.01 & 0.48 & 0.36 & 0.20 & -0.14 \\
\hline EPLT-109 & 0.26 & -0.02 & -0.26 & $-5.985 * *$ & -0.21 & $-1.03 * *$ \\
\hline $\mathrm{SC}_{5} 2-2-1$ & -1.04 & $0.07^{* *}$ & $1.51 * *$ & -1.12 & -0.13 & 0.18 \\
\hline $\mathrm{SC}_{5} 9-3$ & $2.53 * *$ & $0.12 * *$ & $1.18 * *$ & -0.89 & 0.12 & 0.13 \\
\hline SG27-77 & $1.94 * *$ & $-0.06 *$ & 0.48 & $2.09 * *$ & 0.31 & $0.471 * *$ \\
\hline SG26-120 & -0.41 & -0.05 & $-1.43 * *$ & $6.13 * *$ & 0.33 & $0.59 * *$ \\
\hline 118 & $-3.54 * *$ & $0.13 * *$ & $2.18 * *$ & $1.79 * *$ & -0.04 & 0.08 \\
\hline 124 & 0.02 & 0.02 & $1.28 * *$ & $4.79 * *$ & -0.29 & $0.83 * *$ \\
\hline 517 & -0.20 & $-0.08 * *$ & $2.84 * *$ & -0.63 & $-0.90 * *$ & $-0.51 * *$ \\
\hline IR 43 & $-3.06 * *$ & $-0.12 * *$ & $1.48 * *$ & $-5.82 * *$ & $-1.21 * *$ & $-0.99 * *$ \\
\hline IR55 & $-2.26 * *$ & $-0.1 * *$ & 0.21 & $-3.68 * *$ & $-1.46 * *$ & $0.39 *$ \\
\hline IR60 & $-3.83 * *$ & -0.01 & -0.03 & $-4.82 * *$ & $-0.95 * *$ & $-0.43 * *$ \\
\hline SE (testers) & 0.54 & 0.03 & 0.36 & 0.58 & 0.18 & 0.16 \\
\hline
\end{tabular}


Table 2 continued..,

\begin{tabular}{|c|c|c|c|c|c|c|}
\hline & PWT & FG & $\mathrm{SF} \%$ & $1000 \mathrm{SW}$ & GY & PDP \\
\hline \multicolumn{7}{|l|}{ LINES } \\
\hline IR 58025A & $-0.37 * *$ & $-12.83 * *$ & -0.40 & $-0.17 * *$ & $-1.68 * *$ & $-3.23 * *$ \\
\hline IR79156A & $-0.35 * *$ & $-17.59 * *$ & $1.35 * *$ & $0.31 * *$ & $-2.42 * *$ & $-4.84 * *$ \\
\hline APMS 6A & $0.51 * *$ & $26.27 * *$ & $1.41 * *$ & $-0.28 * *$ & $1.45 * *$ & $2.69 * *$ \\
\hline PUSA 5A & $-0.08 *$ & $-9.56 * *$ & $-2.26 * *$ & $0.52 * *$ & $0.91 * *$ & $1.91 * *$ \\
\hline CRMS32A & $0.30 * *$ & $13.71 * *$ & -0.09 & $-0.38 * *$ & $1.73 * *$ & $3.48 * *$ \\
\hline SE (lines) & 0.05 & 1.03 & 0.21 & 0.05 & 0.17 & 0.36 \\
\hline \multicolumn{7}{|l|}{ Testers } \\
\hline 1096 & $0.50 * *$ & $28.82 * *$ & 0.31 & $-0.65 * *$ & $1.35 * *$ & $3.00 * *$ \\
\hline 1005 & $0.42 * *$ & $44.25 * *$ & 0.12 & $-2.20 * *$ & $1.34 * *$ & $2.23 * *$ \\
\hline $619-2$ & 0.07 & -4.14 & -0.12 & $0.55 * *$ & $-1.81 * *$ & $-4.06 * *$ \\
\hline $612-1$ & 0.14 & $-8.06 * *$ & $-2.97 * *$ & $1.37 * *$ & 0.15 & 0.98 \\
\hline $611-1$ & $-0.153 *$ & $-21.38 * *$ & $-5.85 * *$ & $2.41 * *$ & $-2.69 * *$ & $-5.53 * *$ \\
\hline GQ-25 & 0.01 & -3.36 & -0.27 & $0.32 * *$ & 0.27 & 0.25 \\
\hline GQ-37-1 & $-0.40 * *$ & $-11.74 * *$ & $-1.93 * *$ & $-1.55 * *$ & 0.09 & 0.83 \\
\hline GQ-70 & $-0.26 * *$ & $16.66^{* *}$ & $1.62 * *$ & $-2.05 * *$ & 0.55 & $2.04 * *$ \\
\hline GQ-120 & $-0.23 * *$ & $-9.52 * *$ & -0.15 & $-0.91 * *$ & $-0.99 * *$ & $-1.93 *$ \\
\hline KMR-3 & -0.06 & $-15.14 * *$ & 0.67 & $1.10 * *$ & $3.99 * *$ & $9.49 * *$ \\
\hline IBL-57 & 0.13 & $13.92 * *$ & $0.96 *$ & $-1.55 * *$ & $1.89 * *$ & $5.03 * *$ \\
\hline BR827-35 & $0.20 * *$ & $-12.71 * *$ & $-1.62 * *$ & $2.86 * *$ & $-1.002 * *$ & $-2.28 * *$ \\
\hline EPLT-109 & $-0.21 * *$ & $-17.04 * *$ & $-3.39 * *$ & $0.70 * *$ & $-4.68 * *$ & $-9.89 * *$ \\
\hline $\mathrm{SC}_{5} 2-2-1$ & $0.15 *$ & $-5.90 * *$ & -0.42 & $0.57 * *$ & $-1.25 * *$ & $-3.25 * *$ \\
\hline $\mathrm{SC}_{5} 9-3$ & $0.37 * *$ & $9.37 * *$ & $0.96 *$ & $0.30 * *$ & $1.00 * *$ & 1.37 \\
\hline SG27-77 & 0.11 & $14.46 * *$ & $2.71 * *$ & $-1.28 * *$ & $5.12 * *$ & $10.27 * *$ \\
\hline SG26-120 & -0.01 & 2.10 & $3.54 * *$ & $-0.68 * *$ & $1.91 * *$ & $4.62 * *$ \\
\hline 118 & $0.24 * *$ & $20.20 * *$ & $2.87 * *$ & $-0.42 * *$ & $0.75 *$ & 0.81 \\
\hline 124 & $0.15 *$ & $7.03 * *$ & $2.73 * *$ & $-0.27 *$ & $1.23 * *$ & $1.96 *$ \\
\hline 517 & 0.11 & $7.41 * *$ & $3.25 * *$ & -0.07 & $0.79 *$ & 0.23 \\
\hline IR 43 & $-0.48 * *$ & $-11.18 * *$ & 0.70 & $-0.51 * *$ & $-2.50 * *$ & $-5.33 * *$ \\
\hline IR55 & $-0.59 * *$ & $-32.34 * *$ & $-2.80 * *$ & $0.93 * *$ & $-3.17 * *$ & $-6.30 * *$ \\
\hline IR60 & $-0.20 * *$ & $-11.72 * *$ & $-0.92 *$ & 0.12 & $-2.34 * *$ & $-4.55 * *$ \\
\hline SE (testers) & 0.11 & 2.20 & 0.45 & 0.11 & 0.36 & 0.76 \\
\hline
\end{tabular}


Table 3. Top five crosses with high sca effects, per se performance and gca effects of parents for grain yield and its components in rice

\begin{tabular}{|c|c|c|c|c|c|}
\hline \multirow[t]{2}{*}{ Character/ Cross } & \multirow{2}{*}{$\begin{array}{l}\text { Mean per } \\
\text { formance }\end{array}$} & \multirow[t]{2}{*}{$s c a$ effect } & \multicolumn{2}{|l|}{$g c a$ effect } & \multirow[t]{2}{*}{$g c a$ status } \\
\hline & & & Female parent & Male parent & \\
\hline \multicolumn{6}{|l|}{$\begin{array}{l}\text { Days to } 50 \% \text { flowering } \\
\text { (earliness) }\end{array}$} \\
\hline PUSA 5A X IR 60 & 93.33 & $-6.40 * *$ & $0.34 * *$ & 1.48 & $\mathrm{~L} \times \mathrm{L}$ \\
\hline CRMS 32A X IR 43 & 102.50 & $-6.62 * *$ & -0.11 & -0.03 & $\mathrm{~L} \times \mathrm{L}$ \\
\hline APMS 6A X 118 & 96.67 & $-6.23 * *$ & $0.63 * *$ & 2.18 & $\mathrm{~L} \times \mathrm{L}$ \\
\hline IR 58025AX BR 827-35 & 94.50 & $-5.29 * *$ & $-0.78 * *$ & 0.48 & $\mathrm{H} \times \mathrm{L}$ \\
\hline IR 58025A X EPLT 109 & 93.83 & $-5.22 * *$ & $-0.78 * *$ & -0.26 & $\mathrm{H} \times \mathrm{L}$ \\
\hline \multicolumn{6}{|l|}{ Plant height (dwarfnenss) } \\
\hline IR 79156A X GQ 25 & 95.26 & $-11.74 * *$ & $1.68 * *$ & $-1.19 * *$ & $\mathrm{~L} \times \mathrm{H}$ \\
\hline IR 79156A X GQ-120 & 100.78 & $-10.92 * *$ & $1.68 * *$ & $3.51 * *$ & $\mathrm{~L} \times \mathrm{L}$ \\
\hline CRMS 32A X IR 43 & 102.67 & $-10.90 * *$ & $-0.61 * *$ & $-5.82 * *$ & $\mathrm{H} \times \mathrm{H}$ \\
\hline PUSA 5A X IR 60 & 89.19 & $-9.87 * *$ & $-2.63 * *$ & $-4.82 * *$ & $\mathrm{H} \times \mathrm{H}$ \\
\hline IR 79156A X $\quad$ EPLT 109 & 94.17 & $-8.04 * *$ & $1.68 * *$ & $-5.98 * *$ & $\mathrm{LxH}$ \\
\hline \multicolumn{6}{|l|}{ Flag leaf length } \\
\hline CRMS 32 A X SC5 9-3 & 46.48 & $5.97 * *$ & $-0.56^{* *}$ & $2.53 * *$ & $\mathrm{~L} \times \mathrm{H}$ \\
\hline CRMS 32 A X IBL-57 & 46.22 & $5.63 * *$ & $-0.56^{* *}$ & $2.61 * *$ & $\mathrm{~L} \times \mathrm{H}$ \\
\hline IR 79156A X GQ-120 & 47.12 & $5.45^{* *}$ & $1.26^{* *}$ & $1.86^{* *}$ & $\mathrm{H} \times \mathrm{H}$ \\
\hline IR 79156A x 1096 & 45.25 & $4.39 * *$ & $1.26^{* *}$ & 1.05 & $\mathrm{H} \times \mathrm{L}$ \\
\hline CRMS 32 A X 118 & 38.53 & $4.09 * *$ & $-0.56^{* *}$ & $-3.54 * *$ & $\mathrm{~L} \times \mathrm{L}$ \\
\hline \multicolumn{6}{|l|}{ Flag leaf width } \\
\hline APMS 6A X 118 & 2.34 & $0.32 * *$ & $0.12 * *$ & $0.13 * *$ & $\mathrm{H} \times \mathrm{H}$ \\
\hline APMS 6A X 619-2 & 2.20 & $0.28 * *$ & $0.12 * *$ & 0.02 & $\mathrm{Hx} \mathrm{L}$ \\
\hline PUSA 5A X GQ 37-1 & 2.10 & $0.26^{* *}$ & 0.00 & 0.05 & $\mathrm{~L} \times \mathrm{L}$ \\
\hline CRMS 32A X 124 & 2.07 & $0.25 * *$ & 0.02 & 0.02 & $\mathrm{~L} \times \mathrm{L}$ \\
\hline CRMS 32A X IBL 57 & 2.01 & $0.24 * *$ & 0.02 & -0.04 & $\mathrm{~L} \times \mathrm{L}$ \\
\hline \multicolumn{6}{|l|}{ Productive tillers per plant } \\
\hline PUSA 5A X 1096 & 14.80 & $2.01 * *$ & 0.02 & $0.88 * *$ & $\mathrm{~L} \times \mathrm{L}$ \\
\hline IR 79156A XKMR 3 & 13.82 & $1.83 * *$ & -0.06 & $0.80 * *$ & $\mathrm{~L} \times \mathrm{L}$ \\
\hline APMS 6A XSC 5 9-3 & 13.40 & $1.70^{* *}$ & 0.33 & 0.12 & $\mathrm{~L} \times \mathrm{L}$ \\
\hline APMS 6A X124 & 12.84 & $1.55^{* *}$ & 0.33 & -0.29 & $\mathrm{~L} \times \mathrm{L}$ \\
\hline CRMS 32A X IR 43 & 11.42 & $1.24 * *$ & 0.14 & $-1.21 * *$ & $\mathrm{~L} \times \mathrm{H}$ \\
\hline \multicolumn{6}{|l|}{ Panicle length } \\
\hline PUSA 5A X SC5 2-2-1 & 27.16 & $1.67 * *$ & $-0.64 * *$ & 0.18 & $\mathrm{~L} \times \mathrm{L}$ \\
\hline IR 58025A XGQ 37-1 & 27.44 & $1.61^{* *}$ & $0.26^{* *}$ & $-0.38^{* *}$ & $\mathrm{H} \times \mathrm{L}$ \\
\hline APMS 6A X GQ 120 & 27.94 & $1.60^{* *}$ & -0.01 & $0.41^{* *}$ & $\mathrm{~L} \times \mathrm{H}$ \\
\hline IR 79156A X GQ 70 & 27.41 & $1.49 * *$ & $0.20 * *$ & -0.23 & $\mathrm{H} \times \mathrm{L}$ \\
\hline IR 58025A X517 & 26.58 & $1.43 * *$ & $0.26 * *$ & $-0.51 * *$ & $\mathrm{H} \times \mathrm{L}$ \\
\hline
\end{tabular}


Table 3. contd...

\begin{tabular}{|c|c|c|c|c|c|}
\hline \multirow[t]{2}{*}{ Character/ Cross } & \multirow{2}{*}{$\begin{array}{l}\text { Mean } \\
\text { performance }\end{array}$} & \multirow[b]{2}{*}{$s c a$ effect } & \multicolumn{2}{|l|}{$g c a$ effect } & \multirow[t]{2}{*}{ gca status } \\
\hline & & & Female parent & Male parent & \\
\hline \multicolumn{6}{|l|}{ Panicle weight } \\
\hline IR 58025A X IR 60 & 4.98 & $1.19 * *$ & $-0.37 * *$ & $-0.20^{* *}$ & $\mathrm{~L} \times \mathrm{L}$ \\
\hline APMS 6A X SC 5 9-3 & 6.35 & $1.10 * *$ & $0.51 * *$ & $0.37 * *$ & $\mathrm{H} \times \mathrm{H}$ \\
\hline APMS 6A X 118 & 6.20 & $1.01 * *$ & $0.51 * *$ & $0.24 * *$ & $\mathrm{H} \times \mathrm{H}$ \\
\hline IR 79156A X SG 27-77 & 5.10 & $0.98 * *$ & $-0.35^{* *}$ & 0.11 & $\mathrm{~L} \times \mathrm{L}$ \\
\hline IR 79156A X GQ 37-1 & 4.52 & $0.91 * *$ & $-0.35^{* *}$ & $-0.40 * *$ & $\mathrm{~L} \times \mathrm{L}$ \\
\hline \multicolumn{6}{|l|}{ Filled grains per panicle } \\
\hline PUSA 5A X 619-2 & 224.97 & $57.75^{* *}$ & $-9.56 * *$ & -4.14 & $\mathrm{~L} \times \mathrm{L}$ \\
\hline APMS 6A X 1005 & 307.00 & $55.57^{* *}$ & $26.27^{* *}$ & $44.25 * *$ & $\mathrm{H} \times \mathrm{H}$ \\
\hline IR 79156A X SG 27-77 & 233.17 & $55.38^{* *}$ & $-17.59 * *$ & $14.46^{* *}$ & $\mathrm{~L} \times \mathrm{H}$ \\
\hline PUSA 5A X 611-1 & 203.53 & $53.56^{* *}$ & $-9.56 * *$ & $-21.38 * *$ & $\mathrm{~L} \times \mathrm{L}$ \\
\hline CRMS 32A X 517 & 254.90 & $52.87^{* *}$ & $13.71^{* *}$ & $7.41^{* *}$ & $\mathrm{H} \times \mathrm{H}$ \\
\hline \multicolumn{6}{|l|}{ Spikelet fertility $\%$} \\
\hline PUSA 5A X $\quad 611-1$ & 81.31 & $7.99 * *$ & $-2.26^{* *}$ & $-5.85^{* *}$ & $\mathrm{~L} \times \mathrm{L}$ \\
\hline CRMS 32A X EPLT-109 & 84.94 & $7.00 * *$ & -0.09 & $-3.39 * *$ & $\mathrm{~L} \times \mathrm{L}$ \\
\hline CRMS 32A XGQ 70 & 89.93 & $6.98 * *$ & -0.09 & $1.62 * *$ & $\mathrm{~L} \times \mathrm{H}$ \\
\hline APMS 6A X 611-1 & 82.94 & $5.95 * *$ & $1.41 * *$ & $-5.85^{* *}$ & $\mathrm{H} \times \mathrm{L}$ \\
\hline IR 58025A X IR 55 & 84.15 & $5.93 * *$ & -0.40 & $-2.80^{* *}$ & $\mathrm{~L} \times \mathrm{L}$ \\
\hline \multicolumn{6}{|l|}{1000 grain weight } \\
\hline APMS 6A X IBL 57 & 22.78 & $3.13 * *$ & $-0.28^{* *}$ & $-1.55^{* *}$ & $\mathrm{~L} \times \mathrm{L}$ \\
\hline PUSA 5A X 517 & 25.03 & $3.09 * *$ & $0.52 * *$ & $-0.07 * *$ & $\mathrm{H} \times \mathrm{L}$ \\
\hline APMS 6A X IR 60 & 23.67 & $2.35 * *$ & $-0.28 * *$ & $0.12 * *$ & $\mathrm{~L} \times \mathrm{H}$ \\
\hline APMS 6A X EPLT 109 & 24.21 & $2.31 * *$ & $-0.28 * *$ & $0.70 * *$ & $\mathrm{~L} \times \mathrm{H}$ \\
\hline CRMS 32A X IR 43 & 21.93 & $2.24 * *$ & $-0.38^{* *}$ & $-0.51 * *$ & $\mathrm{~L} \times \mathrm{L}$ \\
\hline \multicolumn{6}{|l|}{ Grain yield per plant } \\
\hline APMS 6A X GQ 25 & 34.50 & $10.49^{* *}$ & $1.45 * *$ & 0.27 & $\mathrm{H} \times \mathrm{L}$ \\
\hline PUSA 5A X IR 43 & 34.59 & $10.08^{* *}$ & $0.91 * *$ & $-2.50^{* *}$ & $\mathrm{H} \times \mathrm{L}$ \\
\hline PUSA 5A XIR 55 & 31.72 & $7.89 * *$ & $0.91 * *$ & $-3.17 * *$ & $\mathrm{H} \times \mathrm{L}$ \\
\hline APMS 6A X SC5 9-3 & 36.17 & $7.63 * *$ & $1.45 * *$ & $1.00 * *$ & $\mathrm{H} \times \mathrm{H}$ \\
\hline APMS 6A X 1005 & 36.44 & $7.56^{* *}$ & $1.45^{* *}$ & $1.34 * *$ & $\mathrm{H} \times \mathrm{H}$ \\
\hline \multicolumn{6}{|l|}{ Productivity per day } \\
\hline APMS 6A X GQ 25 & 79.32 & $22.03^{* *}$ & $2.69 * *$ & 0.25 & $\mathrm{H} \times \mathrm{L}$ \\
\hline PUSA 5A X IR 43 & 71.12 & $20.18^{* *}$ & $1.91 * *$ & $-5.33 * *$ & $\mathrm{H} \times \mathrm{L}$ \\
\hline PUSA 5A X IR 55 & 66.69 & $16.73^{* *}$ & $1.91 * *$ & $-6.30^{* *}$ & $\mathrm{H} \times \mathrm{L}$ \\
\hline IR 79156A X IBL 57 & 70.84 & $16.29 * *$ & $-4.84 * *$ & $5.03 * *$ & $\mathrm{~L} \times \mathrm{H}$ \\
\hline PUSA 5A X KMR 3 & 63.56 & $16.16^{* *}$ & $1.91 * *$ & $9.49 * *$ & $\mathrm{H} \times \mathrm{H}$ \\
\hline
\end{tabular}

$\mathrm{H}=$ High $g c a$ effect; L=Low $g c a$ effect 